\title{
An Inverse Problem Approach to the Estimation of Volume Change
}

\author{
Martin Schweiger ${ }^{1}$, Oscar Camara-Rey ${ }^{2}$, William R. Crum ${ }^{2}$, Emma Lewis ${ }^{2}$, \\ Julia Schnabel ${ }^{2}$, Simon R. Arridge ${ }^{1}$, Derek L.G. Hill ${ }^{2}$, and Nick Fox ${ }^{3}$ \\ 1 Department of Computer Science, University College London \\ 2 Centre for Medical Image Computing, University College London \\ 3 Dementia Research Centre, Institute of Neurology, University College London
}

\begin{abstract}
We present a new technique for determining structure-bystructure volume changes, using an inverse problem approach. Given a pre-labelled brain and a series of images at different time-points, we generate finite element meshes from the image data, with volume change modelled by means of an unknown coefficient of expansion on a perstructure basis. We can then determine the volume change in each structure of interest using inverse problem optimization techniques. The proposed method has been tested with simulated and clinical data. Results suggest that the presented technique can be seen as an alternative for volume change estimation.
\end{abstract}

\section{Introduction}

There are a wide number of clinical applications where it is desirable to localize structural change from serial imaging, such as dementia, tumour growth and multiple sclerosis. Several approaches have been proposed to estimate such volume changes [1,2,3. The most powerful make use of serial imaging in conjunction with image registration techniques such as the BBSI [1, the SIENA 2] or the VCM [3]. These techniques are mostly based on a voxel-by-voxel analysis, while clinical interest is normally in structure-by-structure changes.

We propose a new technique for determining structure-by-structure volume changes, using an inverse problem approach. Given a pre-labelled brain, and a series of images at different time-points, we generate finite element meshes from the image data, with volume change modelled by means of an unknown coefficient of expansion on a per-structure basis. The inverse problem then consists of recovering the coefficients of expansion for each structure which would result in the observed volume changes in the mesh. Therefore, the algorithm directly solves for the unknown volume changes in anatomically relevant structures, rather than using an arbitrary regular voxel array. This approach in due course will enable us to incorporate more anatomical and histological information about the disease.

The method has been applied to the recovery of synthetic deformations simulated by an elasticity forward model, to test its robustness in the presence of background noise and local perturbations. Furthermore, it has been applied to 9 pairs of Alzheimer's disease patient images to identify the rate of brain volume change. 


\section{Inverse Problem}

\subsection{Finite Element Deformation Model}

We use a thermoelastic model of soft tissue deformation to simulate the change in brain structure caused by degenerative diseases such as Alzheimer's disease. Thermoelasticity is a convenient way of inducing structural volume change but it should be noted that the thermal expansion coefficient in this model does not have a physical meaning beyond introducing an initial stress term that leads to a volume displacement field. This avoids the need of more accurate models of tissue thermal distributions such as the bioheat equation [4].

The deformation model employs a linear elastic finite element method [5]. Each element of the generated tetrahedral meshes is assigned a set of elastic material properties represented by the elasticity matrix D. The finite element solver is based on the TOAST package [6] which is freely available ${ }^{1}$. In this paper we consider isotropic elastic deformations, where D is symmetric and can be expressed in terms of two parameters, usually Young's modulus $E$ and Poisson's ratio $\nu$. The elastic coefficients are assumed time-invariant. The deformation of the mesh is induced by assigning an isotropic thermal expansion coefficient $\alpha^{(i)}$ to each element $i$, and simulating a global temperature change $\Delta T$. The resulting isotropic thermal expansion enters the description of elastic deformation in the form of an initial element strain $\varepsilon_{0}^{(i)}=\left\{\alpha^{(i)} \Delta T, \alpha^{(i)} \Delta T, \alpha^{(i)} \Delta T, 0,0,0\right\}$, where the relation between stresses $\sigma$ and strains $\varepsilon$ is given by

$$
\sigma^{(i)}=\mathrm{D}^{(i)}\left(\varepsilon^{(i)}-\varepsilon_{0}^{(i)}\right)+\sigma_{0}^{(i)}
$$

Assembling all element contributions of the mesh leads to the linear system

$$
\mathrm{Ku}+\mathbf{f}+\mathbf{f}^{\prime}=\mathbf{r}, \quad K_{i j}=\int_{V} \mathrm{~B}_{i}^{T} \mathrm{DB}_{j} d V
$$

with stiffness matrix $\mathrm{K}$. In an $n$-noded element, $\mathrm{B}$ is the $6 \times 3 n$ strain displacement matrix $\mathrm{B}=\left\{\mathrm{B}_{i}\right\}$ with

$$
\mathrm{B}_{i}=\left\{\begin{array}{ccc}
\frac{\partial N_{i}}{\partial x} & 0 & 0 \\
0 & \frac{\partial N_{i}}{\partial y} & 0 \\
0 & 0 & \frac{\partial N_{i}}{\partial z} \\
\frac{\partial N_{i}}{\partial y} & \frac{\partial N_{i}}{\partial x} & 0 \\
0 & \frac{\partial N_{i}}{\partial z} & \frac{\partial N_{i}}{\partial y} \\
\frac{\partial N_{i}}{\partial z} & 0 & \frac{\partial N_{i}}{\partial x}
\end{array}\right\}, \quad \mathbf{f}^{\prime}=\int_{V} \mathrm{~B}^{T} \mathrm{D} \varepsilon_{0} d V
$$

given nodal shape functions $N_{i} . \mathbf{f}^{\prime}$ contains the volume forces arising from the initial thermal strain, $\mathbf{f}$ combines all other surface and volume force terms, $\mathbf{r}$ defines explicit displacements, and $\mathbf{u}$ is the vector of nodal displacements.

\footnotetext{
1 http://www.medphys.ucl.ac.uk/ martins/toast/index.html
} 


\subsection{Inverse Model}

We formulate the inverse problem of coefficient reconstruction as follows:

Given two meshes corresponding to a baseline state and a distorted target state, e.g. obtained from segmented patient images at two stages of disease, recover the coefficients of expansion $\alpha^{(i)}, i=1 \ldots m$ in a suitable basis of dimension $m$ that, applied to the baseline state, minimise an error norm of difference between the transformed baseline state and the target state.

In this paper we consider an objective function $\psi$ that defines the square sum of nodal displacements $\mathbf{a}_{i} \rightarrow \overline{\mathbf{a}}_{i}$ between two states,

$$
\psi=\sum_{i}\left|\mathbf{a}_{i}-\overline{\mathbf{a}}_{i}\right|^{2} .
$$

If the mesh representations of the two states are not structurally equivalent, a suitable nodal interpolation of the displacement field must be applied. Given the mesh discretisation, the distribution of coefficients of expansion $\alpha(\mathbf{r})$ can now be represented in the element basis $\alpha^{(i)}$ of the mesh. For the purpose of reconstruction we restrict the search space by the additional assumption that the expansion coefficients be homogeneous or piecewise homogeneous within each of a set of anatomical structures of the brain. The inverse problem thus reduces to finding one coefficient of expansion for each segmented region or subregion. Let $m$ be the number of regions, and $n$ the number of mesh nodes. To recover $\alpha=\left\{\alpha^{(i)}\right\} \in \mathbb{R}^{m}$, we employ an iterative Levenberg-Marquardt solver,

$$
\alpha_{k+1}=\alpha_{k}+\left(\mathrm{J}_{k}^{T} \mathrm{~J}_{k}+\eta \mathbf{l}\right)^{-1}\left(\mathrm{~J}_{k}^{T}\left(\mathbf{a}_{k}-\mathbf{a}_{\mathrm{tgt}}\right)\right),
$$

where $\mathbf{a}_{k}$ and $\mathbf{a}_{\text {tgt }}$ are the nodal positions of the deformed and the target mesh, respectively, and $\mathrm{J} \in \mathbb{R}^{n \times m}$ is the Jacobian matrix $J_{i j}=\frac{\partial u_{i}}{\partial \alpha_{j}}$, and $\eta$ is a trustregion control parameter. Because $m$ is small, J can be calculated by explicit perturbation of the region coefficients.

\section{$3 \quad$ Experiments and Results}

We obtained the labelled reference images identifying brain tissue types of the baseline MR image with the FAST algorithm 7]. From the labelled reference image we generated a surface mesh of the whole brain with the marching cubes algorithm [8]. Volume finite element meshes were then obtained using the NETGEN mesh generation software ${ }^{2}$.

In this paper we present two initial tests of reconstruction of the coefficients of expansion: In the first test, synthetic target images are generated by a forward model of thermoelastic deformation, given distributions of the elastic and

2 http://www.hpfem.jku.at/netgen/ 
thermal coefficients in the volume domain. The reconstruction algorithm is then applied to recover region thermal coefficients from the mesh deformation. The performance of the reconstruction algorithm in the presence of random background noise and localised perturbations is investigated. In the second test, the reconstruction is applied to pairs of clinical MR images obtained from $\mathrm{AD}$ patients at a 12-month interval. Comparison of the true changes with model predictions from the reconstructed thermal coefficients gives an indication of the fidelity of the reconstruction when applied to realistic structural change.

\subsection{Simulated Deformation Recovery}

A finite element brain mesh consisting of 163765 nodes and 868404 4-noded tetrahedral elements, segmented into 5 regions of background, CSF, grey and white matter and hippocampi was generated from MR data. To obtain the deformed target image, piecewise constant elastic and thermal coefficients were applied to each of the regions. The region coefficients are shown in Table 1 .

An axial cross section of the target distribution of thermal coefficient $\alpha$ is shown in Fig. 17). In addition to the homogeneous region coefficients, deformations were generated from two further sets of coefficients, by adding random Gaussian background noise (standard deviation $\sigma=0.03$ ) to the coefficients throughout the domain (Fig. 1 b), and by including a localised perturbation of $\alpha=-0.3$ in the grey matter compartment of a frontal lobe (Fig. 15).

Using the linear elasticity forward model, nodal displacements were calculated for each of the three parameter distributions. The surface of the mesh was assumed to coincide with the inner surface of the skull, and Dirichlet boundary conditions were introduced using a Payne-Irons ("big spring") method to suppress the displacements of boundary nodes. The nodal displacements served as the target data for the inverse solver. For this simulation, the reconstruction assumes correct region boundaries of the baseline mesh, and correct elastic coefficients $E$ and $\nu$. The initial estimate of the thermal coefficients $\alpha$ is zero for all regions. The reconstruction results for the region $\alpha$-values are compared to the target values in the left graph of Fig 2. The imposed boundary condition of fixing the mesh surface imposes a constraint of constant mesh volume on the reconstruction, leading to a non-uniqueness which allows to recover $\alpha$ subject to

Table 1. Elastic material properties (Young's modulus $E \times 10^{3} N M^{-2}$ and Poisson's ratio $\nu$ ) and target coefficients of expansion $(\alpha)$ used for simulating deformation

\begin{tabular}{llll}
\hline region & $E$ & $\nu$ & $\alpha$ \\
\hline background & 1 & 0.45 & 0.000 \\
CSF & 1 & 0.45 & 0.300 \\
grey matter & 8 & 0.45 & -0.050 \\
white matter & 4 & 0.45 & 0.030 \\
hippocampi & 1 & 0.45 & -0.1 \\
\hline
\end{tabular}



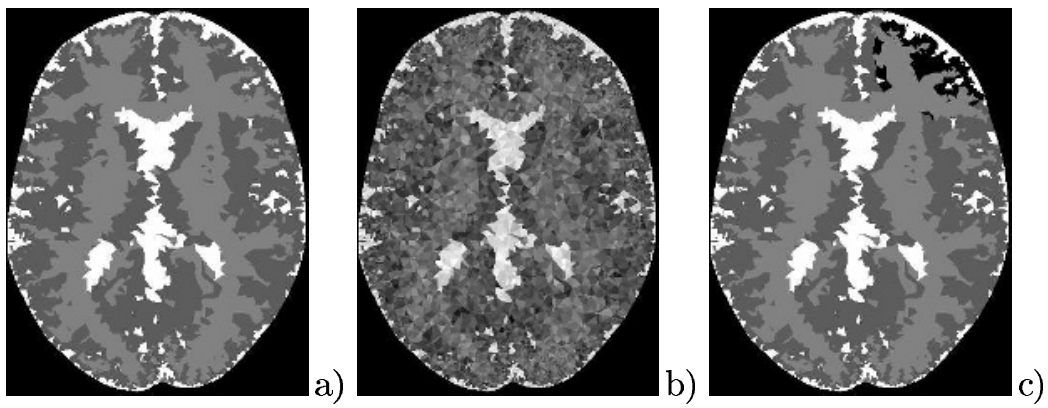

Fig. 1. Axial cross sections of target thermal coefficient distributions. a) piecewise constant region coefficients, b) added random Gaussian background noise, c) added localised grey matter perturbation.
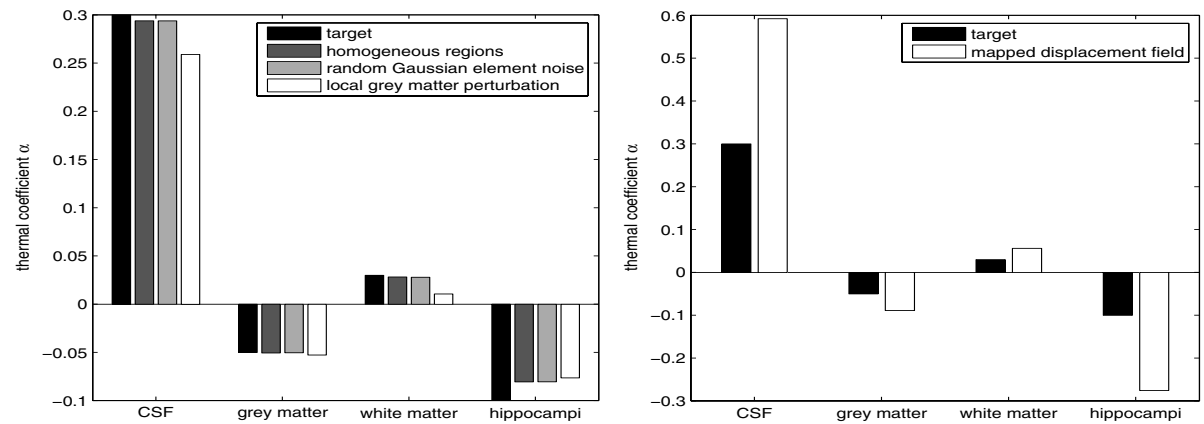

Fig. 2. Left: Reconstructed region thermal coefficients for the 3 distributions shown in Fig. 1 Right: reconstructed coefficients after mapping the displacement field onto a coarse mesh.

an arbitrary global additive term. We take this into account by normalising all results to a value of $\alpha=0$ for the background region.

We find that the reconstruction of homogeneous region parameters provides good quantitative results, and that the addition of Gaussian random noise does not affect the recovered values significantly. The application of a localised perturbation of $\alpha$ has a more significant effect on the recovered region values, because the target distribution is now no longer in the support of the region basis. It is interesting to note that mostly the CSF and white matter regions are affected, but not the grey matter region where the local perturbation was applied.

A further test was performed to investigate the effect of an imperfect knowledge of the region boundaries on the parameter reconstruction, by mapping the nodal displacements calculated for the piecewise homogeneous $\alpha$-distribution (case a) onto a coarser mesh consisting of 10749 nodes and 52297 tetrahedra. The parameter reconstruction was then performed on the coarse mesh, using the interpolated displacement field. The reconstructed region coefficients are shown 
in the right graph of Fig. 2. Due to the misalignment of region boundaries, the target distribution is no longer in the solution space, leading to a degraded quantitative reconstruction. However, the qualitative expansion trends in all regions are still recovered.

\subsection{Clinical Data}

From 9 pairs of Alzheimer MRI images taken at a 12-month interval the corresponding baseline and deformed meshes are generated by using mesh warping techniques [9]. This leads to pairs of meshes with conforming structure and known volumetric differences. In future applications, this method can be replaced by direct mapping of displacement fields between non-conforming meshes. The generated meshes have between 79505 and 138916 elements. Label information is mapped from the reference image into the mesh through a rasterization procedure [10] which computes fractional element labels. To reduce the discretisation error of mapping region boundaries into the mesh, additional boundary regions containing mixed-label elements were introduced, leading to a total of 15 coefficients to be recovered.

Each brain grey-level image at each time-point was registered to the reference image using a 9 degrees of freedom registration ${ }^{3}$ with Normalised Mutual Information as the image similarity measure. Non-rigid fluid registration [11, run at half image resolution was applied. Fluid registration has the advantage for mesh warping purposes that provides diffeomorphic transformations (i.e. they do not fold or tear). After mesh warping based on fluid registrations, we have observed that the geometric quality of the meshes is only slightly reduced (the mean \pm STD aspect ratio $^{4}$ was (a) before mesh warping $=0.67 \pm 0.06$ (b) after mesh warping $=0.66 \pm 0.06$ ), but it does not furnish critical elements (aspect ratio $<0$ ).
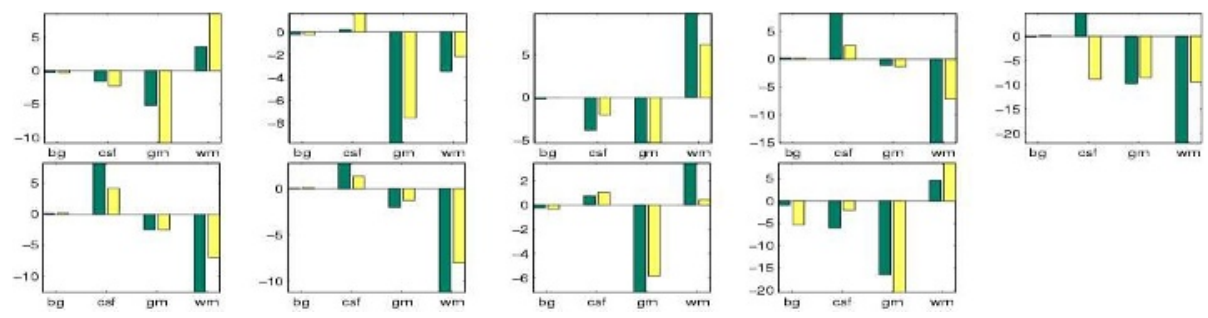

Fig. 3. Comparison between observed and reconstructed volume changes of 4 regions (background, CSF, grey and white matter) in 9 cases

After generating the conforming baseline and target mesh pairs for the 9 cases, the inverse Levenberg-Marquardt solver described in Section 2.2 was used

\footnotetext{
3 http://www.image-registration.com

4 The aspect ratio is defined as the ratio between the longest edge and the radius of the inscribed circle in a tetrahedron, being 1 in the case of optimal quality.
} 
to recover the coefficients of expansion in the 15 generated regions which minimise the nodal displacement between the deformed baseline mesh and the target mesh. In all cases, the reconstructions were started from an initial value $\alpha=0$ in all regions. The element volume changes introduced as a result of the reconstructed deformation were then collected into the 4 segmented regions, using the partial volume information from each element.

Figure 3 shows a comparison of volume changes between baseline and target (dark bars) and between baseline and reconstruction (bright bars) for all 9 cases. It can be seen that the reconstructed deformations predict the trend of volume change correctly in the majority of cases, although the absolute values are not always agreeing well. The results show that the thermoelastic deformation model has a potential to describe the types of structure change seen in AD, but that a further subdivision of the anatomical structure may be required to provide a sufficiently large search space for accurate representation of the deformation in the basis of the inverse solver.

\section{Discussion and Conclusion}

The method of recovering coefficients of expansion from a baseline and a deformed mesh has been shown to converge robustly in simulated data generated from piecewise homogeneous region parameters even in the presence of random zero-mean background noise and localised parameter perturbations. Where deformations were applied that could not be represented in the low-dimensional basis of homogeneous region coefficients, a qualitative recovery of trends within the regions is still feasible, but the quantitation of region parameters deteriorates. In that case, the convergence of the inverse solver could be improved by further subdivision of the segmented regions and increasing the dimension of the parameter solution space, for example by a segmentation into anatomical sub-regions using information from manually segmented atlases that separate structures likely to undergo structural changes in the course of disease. This will maintain an anatomical interpretation of the deformation coefficients and allow to easily distinguish between specific patterns of different types of dementia.

The proposed technique has also been applied to a set of 9 pairs of images from AD patients. Despite the restriction to a low-dimensional solution space of 15 regions the reconstruction results show good qualitative agreement of region volume change when compared with target data in the majority of cases. As with the simulated data set, further improvement can be expected by increasing the dimension of the search space. One strategy of parameter space refinement that retains the correspondance with anatomical features would be a subdivision of tissue types into separate lobes of the brain.

The results presented in this paper indicate that a region-based approach of structure change can be seen as a feasible alternative to other volume change estimation techniques. By representing the coefficients of deformation in a basis that is directly associated with the underlying anatomical structure, the recovered expansion parameters can be interpreted more immediately than in a 
voxel-based technique in terms of anatomical change, such as grey-matter volume loss. A further subdivision of the basis representation into regions of interest may be suitable to recover disease-specific parameters and so aid in the diagnosis of dementia. Future work will also be focused on a rigorous validation of the presented technique, by comparing its performance with classical volume change measurement methods such as the BBSI or the VCM.

\section{Acknowledgments}

The authors acknowledge grant support from the Engineering and Physical Sciences Research Council, grants GR/N148/01 and GR/S48837/02, and from the Medical Research Council.

\section{References}

1. Freeborough, P.A., Fox, N.C.: The boundary shift integral: an accurate and robust measure of cerebral volume changes from registered repeat MRI, IEEE Transactions on Medical Imaging, 16 (5) (1997) 623-629

2. Smith, S.M., De Stefano, N., Jenkinson, M., Matthews, P.M.: Normalized accurate measurement of longitudinal brain change, Journal of Computer Assisted Tomography, 25 (3) (2001) 466-475

3. Fox, N.C., Crum, W.R., Scahill, R.I., Stevens, J.M., Janssen, J.C., Rossor, M.N.: Imaging of onset and progression of Alzheimer's disease with voxel-compression mapping of serial magnetic resonance images, Lancet, 358 (9277) (2001) 201-205

4. Durkee, J.W, Antich, P.P.: Exact solutions to the multi-region time-dependent bioheat equation with transient heat sources and boundary conditions, Phys. Med. Biol, 36 (3) (1991) 345-368

5. Zienkiewicz, O.C., Taylor, R.L.: The Finite Element Method. 4th edn. McGrawHill, London (1987)

6. Schweiger, M., Arridge, S.R.: Image reconstruction in optical tomography using local basis functions. Journal of Electronic Imaging 12 (2003) 583-593

7. Zhang, Y., Brady, M., Smith, S.: Segmentation of brain MR images through a hidden Markov random field model and the expectation maximization algorithm, IEEE Transactions on Medical Imaging, 20 (1) (2001) 45-57

8. Lorensen, W.E., Cline, H.E.: Marching cube, a high resolution 3D surface reconstruction algorithm, Proceedings of SIGGRAPH 1987, 21 (1987) 163-169

9. Castellano-Smith, A.D., Hartkens, T., Schnabel, J.A., Hose D.R., Liu, H. , Hall, W.A., Truwit, C.L., Hawkes, D.J., Hill, D.L.G.: A registration based mesh construction technique for finite element models of brains. Proceedings of SPIE Medical Imaging 2002, volume 4683, 538-549

10. Sermesant, M., Forest, C., Pennec, X., Delingette, H., Ayache, N.: Deformable biomechanical models: Application to $4 \mathrm{D}$ cardiac image analysis, Medical Image Analysis, 7 (4) (2003) 475-488

11. Crum, W.R., Rueckert, D., Jenkinson, M., Kennedy, D., Smith, S.M.: A Framework For Detailed Objective Comparison of Non-Rigid Registration Algorithms in Neuroimaging, Proceedings of MICCAI 2004, LNCS 3216, 679-686 